\title{
Tunable coupling of transmission-line microwave resonators mediated by an rf SQUID
}

Friedrich Wulschner ${ }^{1,2}$, Jan Goetz ${ }^{1,2}$, Fabian R Koessel ${ }^{1,2}$, Elisabeth Hoffmann ${ }^{1,2}$, Alexander Baust ${ }^{1,2,3}$, Peter Eder 1,2,3, Michael Fischer 1,2,3, Max Haeberlein 1,2, Manuel J Schwarz ${ }^{1,2,3}$, Matthias Pernpeintner ${ }^{1,2,3}$, Edwar Xie ${ }^{1,2,3}$, Ling Zhong ${ }^{1,2,3}$, Christoph W Zollitsch ${ }^{1,2}$, Borja Peropadre ${ }^{4}$, Juan-Jose Garcia Ripoll15, Enrique Solano $2,6,7$, Kirill G Fedorov ${ }^{1,2}$, Edwin P Menzel ${ }^{1,2}$, Frank Deppe ${ }^{1,2,3}$ (D) Achim Marx ${ }^{1}$ and Rudolf Gross ${ }^{1,2,3^{*}}$ (D)

"Correspondence:

Rudolf.Gross@wmi.badw.de

'Walther-Meißner-Institut, Bayerische Akademie der

Wissenschaften, Garching, 85748 Germany

${ }^{2}$ Physik-Department, Technische Universität München, Garching, 85748, Germany

Full list of author information is available at the end of the article

\begin{abstract}
We realize tunable coupling between two superconducting transmission line resonators. The coupling is mediated by a non-hysteretic rf SQUID acting as a flux-tunable mutual inductance between the resonators. We present a spectroscopic characterization of the device. In particular, we observe couplings $g / 2 \pi$ ranging between $-320 \mathrm{MHz}$ and $37 \mathrm{MHz}$. In the case of $g \simeq 0$, the microwave power cross transmission between the two resonators is reduced by almost four orders of magnitude as compared to the case where the coupling is switched on.
\end{abstract}

\section{Introduction}

In circuit quantum electrodynamics, the controllable interaction of circuit elements is a highly desirable resource for quantum computation and quantum simulation experiments. The most common method is a static capacitive or inductive coupling between cavities and/or qubits. In such a system, exchange of excitations can be controlled by either tuning the circuit elements in and out of resonance or using sideband transitions [1-4]. While this approach has proven to be useful for few coupled circuit elements, it seems impracticable for larger systems, where it is hard to provide sufficient detunings between all circuit elements [5]. Therefore, one may alternatively use tunable coupling elements such as qubits [6-9] or SQUIDs [10-15]. One particular example for an interesting application of such actively coupled circuit elements are quantum simulations of bosonic many-body Hamiltonians [16-20]. In such a scenario, the bosonic degrees of freedom can be represented by networks of (possibly nonlinear) superconducting resonators. For this quantum simulator, a tunable coupler would constitute an important control knob. A more general scope of this device is the controllable routing of photonic states on a chip, which is interesting for quantum information as well as quantum simulation experiments.

In this work, we experimentally investigate the case of two nearly frequency-degenerate superconducting transmission line resonators coupled by an rf SQUID acting as a tunable mutual inductance in the spirit of Refs. [21-23]. Although such a setup looks similar

(c) 2016 Wulschner et al. This article is distributed under the terms of the Creative Commons Attribution 4.0 International License (http://creativecommons.org/licenses/by/4.0/), which permits unrestricted use, distribution, and reproduction in any medium, provided you give appropriate credit to the original author(s) and the source, provide a link to the Creative Commons license, and indicate if changes were made. 
to the case of a flux qubit mediated coupling [6], there are important conceptual differences resulting in performance advantages. In a flux qubit coupler [24, 25], the resonatorresonator coupling is limited to twice the dispersive qubit-resonator shift (typically a few $\mathrm{MHz}$ ). Efforts to increase the maximum coupling by relaxing the dispersive coupling assumption have contributed to the limited isolation of $2.6 \mathrm{~dB}$ between the resonators in the off-state of the coupler in Ref. [6]. This conceptual disadvantage obviously outweighs the potential quantum switch properties $[24,25]$ of the flux qubit coupler for many practical applications. In contrast, couplers between superconducting qubits based on the classical phase dynamics of an rf SQUID have shown large couplings $[11,13,14]$ and good isolation properties [14]. Compared to our previous work [6], we achieve two significant improvements: First, the range of achievable coupling strengths between the resonators is increased from $g /(2 \pi) \in[-28.7 \mathrm{MHz}, 8.4 \mathrm{MHz}]$ to $g /(2 \pi) \in[-302 \mathrm{MHz}, 37 \mathrm{MHz}]$. Second, comparing the signal transmission between both resonators for the coupled $(g \gg 0)$ and decoupled $(g \simeq 0$ ) case, the signal isolation is increased from $2.6 \mathrm{~dB}$ to $38.5 \mathrm{~dB}$. Especially the increased isolation of the device discussed in the present work is a key prerequisite for several applications both in quantum simulation and quantum computation setups. The manuscript is structured as follows. After briefly discussing the relevant theory in Section 2 we introduce the sample and measurement setup in Section 3. In Section 4, we present a spectroscopic characterization of the rf SQUID coupler followed by a summary and conclusions in Section 5. The appendix contains a short discussion of the additional feature of parametric amplification observed in our device.

\section{System Hamiltonian}

An optical micrograph of the sample is shown in Figure 1(a). Our system is comprised of an rf SQUID galvanically coupled to the center conductor of two coplanar stripline resonators. These resonators, A and B, can be described as quantum harmonic oscillators using the Hamiltonian

$$
H_{\text {res }}=\hbar \omega_{\mathrm{A}} \hat{a}^{\dagger} \hat{a}+\hbar \omega_{\mathrm{B}} \hat{b}^{\dagger} \hat{b}
$$

(a)

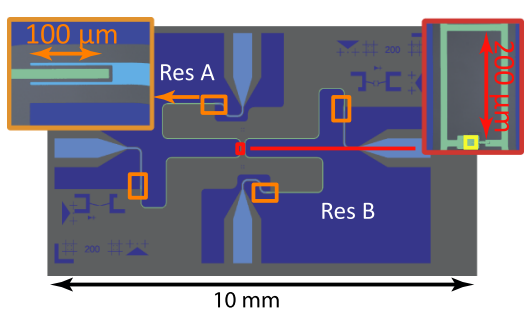

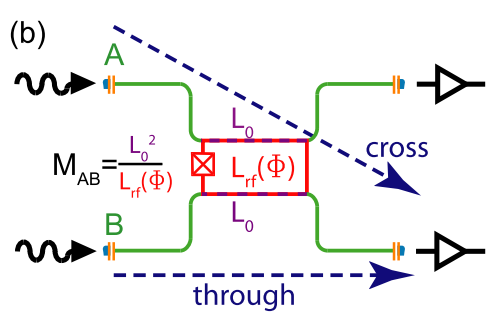

Figure 1 Sample and measurement setup. (a) Sketch of the sample chip. Dark blue: Resonator groundplanes. Green: Resonator center conductors. Light blue: Feed line center conductors. The insets are (false color) optical micrographs of the coupling capacitors (orange), the rf SQUID (red), and the rf SQUID junction (yellow). (b) Operating principle of the device: Both resonators share an inductance $L_{0}$ (purple) with the SQUID and the SQUID itself can be treated as an effective inductance $L_{\mathrm{rf}}(\Phi)$ (red, see Equation (4)), resulting in an effective mutual inductance of $M_{\mathrm{AB}}=L_{0}^{2} / L_{\mathrm{rf}}(\Phi)$ between both resonators (see Equation (7)). The sketched measurement setup contains attenuated input lines (indicated by wiggly arrows), output lines including cryogenic and room temperature microwave amplifiers (triangular symbols), and possible measurement paths (blue dashed arrows). 
Here, $\omega_{\mathrm{A}}$ and $\omega_{\mathrm{B}}$ are the resonance frequencies and $\hat{a}^{\dagger}, \hat{b}^{\dagger}, \hat{a}$, and $\hat{b}$ are the bosonic creation and annihilation operators. The effect of the rf SQUID on the system properties can be modeled in terms of an effective inductance [23, 26, 27]. The fluxes $\Phi_{\mathrm{A}}$ and $\Phi_{\mathrm{B}}$ generated by the resonators in the rf SQUID give rise to an inductive interaction energy. The rf SQUID consists of a superconducting loop with inductance $L_{\mathrm{s}}$, which is interrupted by a Josephson junction with critical current $I_{\mathrm{c}}$. The flux $\Phi$ threading the SQUID loop gives rise to a circulating current

$$
I_{\mathrm{S}}(\Phi)=-I_{\mathrm{c}} \sin \left(2 \pi \Phi / \Phi_{0}\right),
$$

where $\Phi_{0}$ is the flux quantum. Here, $\Phi$ is the sum of the externally applied flux $\Phi_{\text {ext }}$ and the flux generated by $I_{\mathrm{s}}$,

$$
\Phi=\Phi_{\text {ext }}+L_{\mathrm{s}} I_{\mathrm{s}}(\Phi)
$$

Since, in the experiment, the screening parameter $\beta=2 \pi L_{\mathrm{s}} I_{\mathrm{c}} / \Phi_{0}<1$, the dependence of the total flux on the external flux is single-valued. From Equation (2) and Equation (3), an expression for the effective SQUID inductance with respect to external fluxes is obtained,

$$
\frac{1}{L_{\mathrm{rf}}(\Phi)}=\frac{\partial I_{\mathrm{s}}}{\partial \Phi_{\mathrm{ext}}}=-\frac{1}{L_{\mathrm{s}}} \frac{\beta \cos \left(2 \pi \frac{\Phi}{\Phi_{0}}\right)}{1+\beta \cos \left(2 \pi \frac{\Phi}{\Phi_{0}}\right)} .
$$

For the flux $\Phi_{\mathrm{A}, \mathrm{B}}$, generated by the resonators A, B respectively, in the limit $\Phi_{\mathrm{A}, \mathrm{B}} \ll \Phi_{0}$, one can write the change in SQUID energy caused by the resonator fluxes as $[13,21,26$, $28-30]$

$$
H_{\text {ind }}=\frac{\left(\Phi_{\mathrm{A}}-\Phi_{\mathrm{B}}\right)^{2}}{2 L_{\mathrm{rf}}(\Phi)}=\frac{\Phi_{\mathrm{A}}^{2}+\Phi_{\mathrm{B}}^{2}-2 \Phi_{\mathrm{A}} \Phi_{\mathrm{B}}}{2 L_{\mathrm{rf}}(\Phi)}
$$

Inserting the fluxes generated by the resonators [31] in Equation (5), two essential properties of the system become obvious. First, the $\Phi_{\mathrm{A}, \mathrm{B}}^{2}$-terms in Equation (5) lead to dressed resonator frequencies

$$
\tilde{\omega}_{\mathrm{A}, \mathrm{B}}=\omega_{\mathrm{A}, \mathrm{B}} \sqrt{1-2 \frac{L_{0}^{2}}{L_{\mathrm{A}, \mathrm{B}} L_{\mathrm{rf}}(\Phi)}} \approx \omega_{\mathrm{A}, \mathrm{B}}\left(1-\frac{L_{0}^{2}}{L_{\mathrm{A}, \mathrm{B}} L_{\mathrm{rf}}(\Phi)}\right),
$$

where $L_{\mathrm{A}, \mathrm{B}}$ is the inductance of the resonators and $L_{0}$ the inductance of the segment shared between resonator and rf SQUID. The second effect of Equation (5), caused by the term $\propto \Phi_{\mathrm{A}} \Phi_{\mathrm{B}}$, is a flux dependent coupling

$$
g_{\mathrm{AB}}(\Phi)=-\underbrace{\sqrt{\frac{\omega_{\mathrm{A}}}{L_{\mathrm{A}}}}}_{I_{\mathrm{A}}} \underbrace{\sqrt{\frac{\omega_{\mathrm{B}}}{L_{\mathrm{B}}}}}_{I_{\mathrm{B}}} \underbrace{\frac{L_{0}^{2}}{L_{\mathrm{Lf}}(\Phi)}}_{M_{\mathrm{AB}}}
$$

between the resonators. As indicated in Equation (7) the coupling can be seen as the product of the resonator vacuum currents $\left(I_{\mathrm{A}}, I_{\mathrm{B}}\right)$ at the SQUID position with the effective second order mutual inductance $M_{\mathrm{AB}}=L_{0}^{2} / L_{\mathrm{rf}}(\Phi)$ mediated by the SQUID. Due to their 
vicinity on the chip, the two resonators also induce directly currents into each other, resulting in a flux independent direct inductive coupling component $g_{\mathrm{I}}$ between the resonators. Thus the total coupling reads

$$
g(\Phi)=g_{\mathrm{AB}}(\Phi)+g_{\mathrm{I}}
$$

Equation (4) shows that $g_{\mathrm{AB}}(\Phi)$ can be positive or negative depending on the applied flux. By applying a suitable flux, the rf SQUID mediated coupling compensates the direct inductive coupling. In this way, one can turn on and off the net coupling between the resonators. After a rotating wave approximation the full Hamiltonian reads

$$
H=\hbar\left(\hat{a}^{\dagger} \hat{b}^{\dagger}\right)\left(\begin{array}{cc}
\tilde{\omega}_{\mathrm{A}} & g(\Phi) \\
g(\Phi) & \tilde{\omega}_{\mathrm{B}}
\end{array}\right)\left(\begin{array}{l}
\hat{a} \\
\hat{b}
\end{array}\right) .
$$

The eigenvalues of Equation (9) correspond to the new eigenfrequencies

$$
\Omega_{1,2}=\frac{\tilde{\omega}_{\mathrm{A}}+\tilde{\omega}_{\mathrm{B}}}{2} \pm \sqrt{g(\Phi)^{2}+\frac{\left(\tilde{\omega}_{\mathrm{A}}-\tilde{\omega}_{\mathrm{B}}\right)^{2}}{4}} .
$$

\section{Sample and measurement setup}

Figure 1(a) shows the layout of the sample chip. In the resonator design, we omit the second groundplane to reduce the direct geometric coupling between the two resonators. The rf SQUID is galvanically connected to both center strips of the resonators over a length of $200 \mu \mathrm{m}$. The sample is fabricated as follows. First, a $100 \mathrm{~nm}$ thick niobium layer is sputter deposited onto a $250 \mu \mathrm{m}$ thick, thermally oxidized silicon wafer. The resonators and the SQUID loop are patterned using optical lithography and reactive ion etching. The Josephson junction of the SQUID is fabricated in a $\mathrm{Nb} / \mathrm{AlO}_{\mathrm{x}} / \mathrm{Nb}$ trilayer process with $\mathrm{SiO}_{2}$ as insulating layer between top and bottom electrode [32]. ${ }^{\mathrm{a}}$ The resonators have a characteristic impedance of $Z_{0}=64 \Omega$ and the resonance frequencies $\omega_{\mathrm{A}} / 2 \pi=6.482 \mathrm{GHz}$ and $\omega_{\mathrm{B}} / 2 \pi=6.461 \mathrm{GHz}$. ${ }^{\mathrm{b}}$ The SQUID loop has dimensions of $200 \mu \mathrm{m} \times 100 \mu \mathrm{m}$ and a screening parameter $\beta=0.934$ to maximize the coupling according to Equation (4) while keeping the SQUID monostable. The sample is mounted inside a gold plated copper box, which is attached to the base temperature stage of a dilution refrigerator operating at $26 \mathrm{mK}$. A superconducting solenoid attached to the top of the sample box is used to generate the external flux applied to the rf SQUID. As depicted in Figure 1(b), one port of each resonator is connected to an attenuated input microwave line, whereas the remaining ports are connected to output lines containing microwave amplifiers.

\section{Resonator spectroscopy}

We first extract the properties of the rf SQUID coupler from transmission measurements through the individual resonators. As indicated in Figure 1, we call this type of measurement a "through-measurement." In contrast, in the "cross-measurements" we inject a signal into one of the resonators and probe the output of the other one. Based on the configuration of our input line, we estimate an average photon number of approximately one in the resonators for these measurements. In Figure 2(a) and Figure 2(b), the through measurements of resonator $\mathrm{A}$ and $\mathrm{B}$ are shown depending on the applied flux $\Phi_{\text {ext }}$. According to Equation (4) and Equation (5), the modulation of the resonator 
(a)

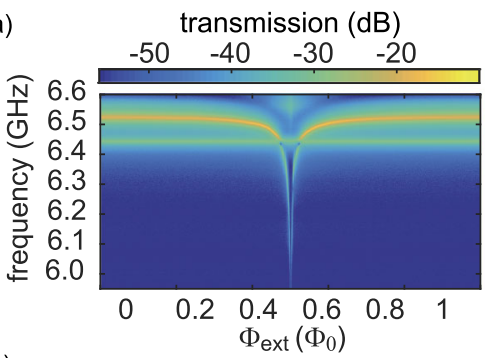

(c)

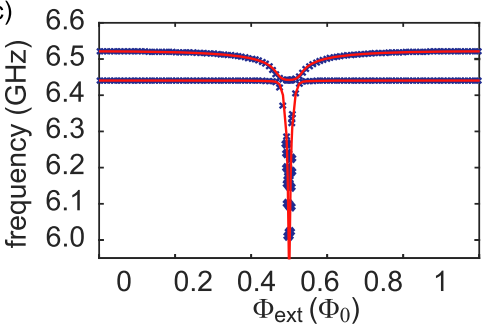

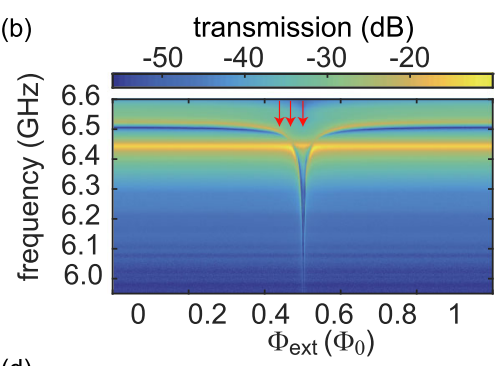

(d)

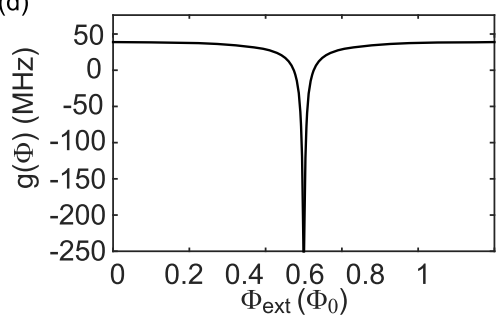

Figure 2 Uncalibrated through transmission magnitude (color coded) as a function of probe frequency and externally applied flux for (a) resonator $\mathbf{A}$ and $(\mathbf{b})$ resonator $\mathbf{B}$. The red arrows mark the flux values, for which transmission vs. frequency cuts are shown in Figure 4. (c) Fit (red line) of Equation (10) to the extracted center frequencies (crosses). The modes are taken from the through measurements, where they are more pronounced. This is especially necessary when the coupling is smaller than the detuning of the resonators. (d) Plot of the coupling rate $g(\Phi)$ between both resonators obtained by the fit in (c).

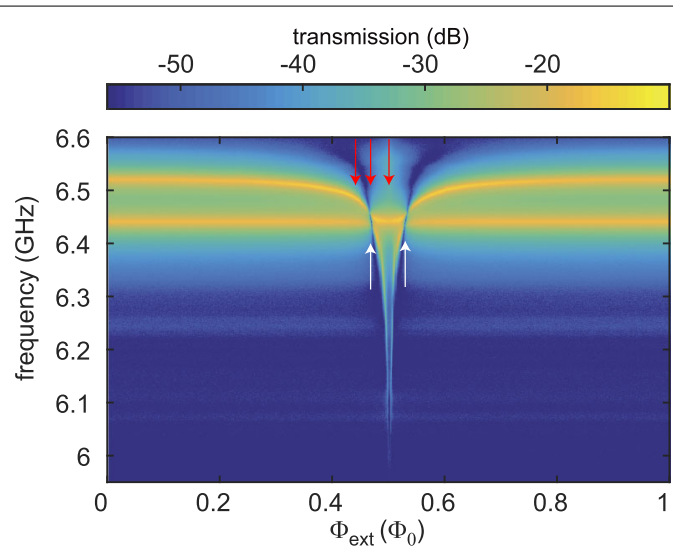

Figure 3 Uncalibrated cross-transmission from resonator $A$ to resonator $B$ as a function of the applied flux. Near $\Phi_{\text {ext }}=0.468 \Phi_{0}$ and $\Phi_{\text {ext }}=0.532 \Phi_{0}$ (white arrows), the resonators decouple and signal transmission is blocked. The red arrows mark the flux values, for which transmission vs. frequency cuts are shown in Figure 4

modes due to the presence of the $\operatorname{rf~SQUID~is~} \Phi_{0}$-periodic and symmetric with respect to $\Phi_{\text {ext }}=\Phi_{0} / 2$. The two modes of Equation (10) manifest themselves as two resonances in the spectroscopy data. As expected, we observe a flux dependent mode distance, caused by the flux tunable mutual inductance of the rf SQUID. For most flux values, one observes two resonance peaks independent of the chosen input and output port. However near $\Phi_{\text {ext }}=0.468 \Phi_{0}$ and $\Phi_{\text {ext }}=0.532 \Phi_{0}$, only a single peak is present in the through measurements and cross transmission is strongly suppressed (see Figure 3). These are the points where the SQUID-mediated coupling compensates the direct inductive coupling resulting in a vanishing total coupling and, hence, completely decoupled resonators. 
Note that for $g(\Phi)=0$, the Hamiltonian of Equation (9) becomes diagonal and each of the two modes reflects the excitation of one of the resonators. In Figure 2(c), the center frequencies of the normal modes $\Omega_{1,2}$ derived from the data in Figure 2(a) and Figure 2(b) are plotted along with a fit using Equation (10). From this fit, we further calculate the flux-dependent coupling rate $g(\Phi)$ between the resonators. The result is shown in Figure 2(d). Upon closer inspection of Figure 2(c), we find a minimum distance on the order of $20 \mathrm{MHz}$ between these modes. This finite gap is caused by a small detuning $\Delta=\omega_{\mathrm{A}}-\omega_{\mathrm{B}}=2 \pi \times 21.3 \mathrm{MHz}$ of the resonators. We also observe different decay rates of the resonators, which we extract from the through measurements of both resonators at a decoupling point. Lorentzian fits lead to $\gamma_{\mathrm{A}} / 2 \pi=3.6 \mathrm{MHz}$ and $\gamma_{\mathrm{B}} / 2 \pi=6.1 \mathrm{MHz}$. Based on our experience with $\mathrm{Nb}$ resonators without a SQUID junction [33, 34], our device is in the overcoupled regime where losses are dominated by the coupling capacitors. The fact that resonator A has a smaller linewidth and a slightly higher eigenfrequency than resonator $B$ indicates a smaller effective coupling capacitance [35]. We attribute this observation to fabrication or sample contacting imperfections. Therefore, we assume $L_{\mathrm{A}} / L_{\mathrm{B}} \approx 1$. Furthermore, we define the fitting parameter $g_{0}=\sqrt{\omega_{\mathrm{A}} \omega_{\mathrm{B}}} L_{0}^{2} /\left(\sqrt{L_{\mathrm{A}} L_{\mathrm{B}}} L_{\mathrm{s}}\right)$. In this way, the rf SQUID coupling reads $g_{\mathrm{AB}}=g_{0} \beta \cos \left(2 \pi \frac{\Phi}{\Phi_{0}}\right) /\left[1+\beta \cos \left(2 \pi \frac{\Phi}{\Phi_{0}}\right)\right]$. Fitting Equation (10) to the data as shown in Figure 2(c), we obtain $\omega_{\mathrm{A}} / 2 \pi=6.482 \mathrm{GHz}, \omega_{\mathrm{B}} / 2 \pi=6.461 \mathrm{GHz}, \beta=0.934$, $g_{\mathrm{I}} / 2 \pi=29.0 \mathrm{MHz}$ and $g_{0} / 2 \pi=20.4 \mathrm{MHz}$. From the mode distance we find $g(\Phi) / 2 \pi$ ranging between $37 \mathrm{MHz}$ and $-320 \mathrm{MHz}$. Beyond this value, the lower mode becomes too broad due to its steep flux dependence.

Next, we analyze the properties of our device in the coupled and decoupled state in more detail. Because of the small detuning of the resonators, the coupled modes are not necessarily symmetric and antisymmetric superpositions of the uncoupled modes. This is also seen in the spectroscopy of the single resonators (see Figure 2), where the modes have different intensities. The mode mixing can be estimated from the eigenvectors of the Hamiltonian in Equation (9). For $g / 2 \pi=37 \mathrm{MHz}$ and $g / 2 \pi=-320 \mathrm{MHz}$ we obtain the mixing ratios $63: 37$ and $52: 48$, respectively. Hence, in the latter case, our sample satisfies the condition $|g| \gg|\Delta|$, where the detuning becomes insignificant. In the decoupled case near $\Phi_{\text {ext }}=0.468 \Phi_{0}$ and $\Phi_{\text {ext }}=0.532 \Phi_{0}$, the off-diagonal elements in the Hamiltonian of Equation (9) vanish and the modes are pure excitations of resonator A or B.

In this situation, it is particularly instructive to examine the cross-transmission spectra such as the one shown in Figure 3, where resonator A is driven and resonator B is probed. Here, we clearly see that in two narrow regions around $\Phi_{\text {ext }}=0.468 \Phi_{0}$ and $\Phi_{\text {ext }}=0.532 \Phi_{0}$, where the net coupling $g(\Phi)$ approaches zero, the microwave transmission between the resonators is blocked. We gain further insight by comparing the throughand cross-transmission spectra in Figure 4(a) and Figure 4(b). For $|g(\Phi)| \gg|\Delta|$ [see Figure 4(a)], both measurements exhibit similar peak heights. Since both measurements use the same output line, we relate the small difference of approximately $1.5 \mathrm{~dB}$ mainly to the slightly different losses in the input lines. For $g(\Phi) \approx 0$, however, the cross-transmission is suppressed by $40 \mathrm{~dB}$ on resonance as shown in Figure 4(b), corresponding to a relative transmission change of $38.5 \mathrm{~dB}$. This result confirms that we can sufficiently compensate the direct inductive coupling with the tunable SQUID-mediated coupling. Finally, in Figure 4(c) we show the transmission for a flux value, where $g(\Phi)$ and $\Delta$ are comparable. In the through measurement, the detuning manifests itself in the form of unequal peak 

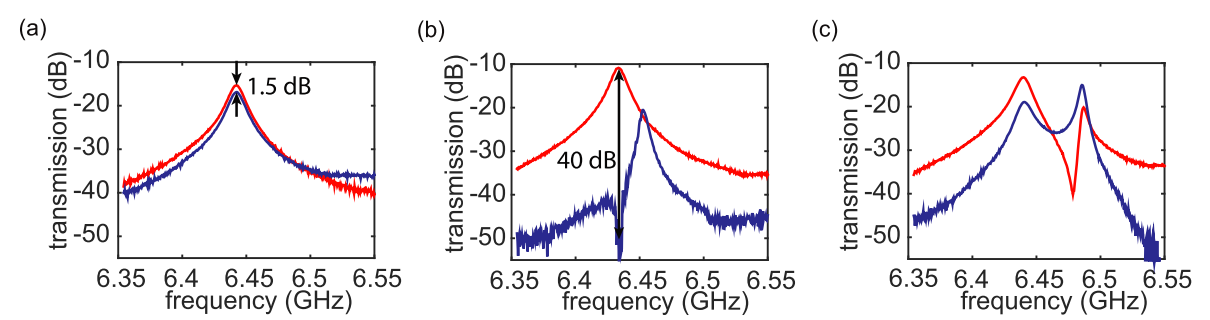

Figure 4 Uncalibrated through- (red) and cross-measurements (blue), obtained for three different flux values. (a) $\Phi_{\text {ext }}=\Phi_{0} / 2,|g| \gg|\Delta|$ : strongly coupled regime, only the mode $\Omega_{1}$ is shown.

(b) $\Phi_{\text {ext }}=0.468 \Phi_{0} / 2,|g| \simeq 0$ : decoupling point. (c) $\Phi_{\text {ext }}=0.439 \Phi_{0} / 2,|g| \simeq \Delta$.

heights and an anti-resonance dip, which is not centered between the resonance peaks $[36,37]$.

\section{Conclusions}

In conclusion, we present a flux-tunable coupling between two superconducting resonators based on a SQUID containing a single Josephson junction. Spectroscopically, we measure negative and positive couplings ranging from $-320 \mathrm{MHz}$ to $37 \mathrm{MHz}$. Furthermore, the observed suppression of the cross-transmission of up to $38.5 \mathrm{~dB}$ proves the ability to effectively turn off the coupling and is an important improvement over previous work [6], where still $27.5 \%$ ( $2.6 \mathrm{~dB}$ change in cross-transmission) of the signalpower was transmitted to the uncoupled resonator for $g \simeq 0$. With the achieved performance, our coupler can be considered as a useful tool for quantum computation with a controlled nearest neighbor interaction or to route information on a chip in a controllable way. Regarding quantum simulation experiments [16-19, 38, 39], our device could be especially useful because it allows one to change both amplitude and sign of the coupling constant. For such future experiments, a fast flux antenna enabling a non-adiabatic change of the coupling strength can be integrated onto the chip straightforwardly.

\section{Appendix}

So far, we have controlled the inductance of the rf SQUID and therefore the coupling between the two resonators using a quasistatic flux bias through the SQUID loop. When additionally modulating this inductance at suitable microwave frequencies, one expects parametric amplification. In contrast to traveling-wave parametric amplifiers, where the nonlinear medium extends over a length comparable to the wavelength (e.g., many SQUIDs in a transmission line) [40-42], our setup belongs to a class of experiments where a single SQUID couples to a resonant circuit [15, 43-47]. Although this approach suffers from bandwidth and dynamic range limitations, it requires only a single Josephson junction and thereby reduces fabrication complexity to a minimum, as it is required for scalable architectures. Specifically, we exploit the flux-dependent resonance frequency of the $\Omega_{1}$ mode and operate our device as a flux-driven parametric amplifier by applying an additional drive tone at $\omega_{\mathrm{D}}=2 \Omega_{1}$ to the input line. The drive tone periodically modulates the flux threading the SQUID loop and therefore the mode frequency, leading to parametric amplification. To characterize the performance of our device, we calculate the power gain $(G)$ as well as the bandwidth characterized by the full width half maximum $\Delta \Omega$ of the amplified signals. Figure 5 shows the transmission in the vicinity of 
Figure 5 Uncalibrated through-transmission vs. frequency around the coupled mode frequency $\Omega_{1}$ for $\Phi_{\text {ext }}=0.450 \Phi_{0}$ and an additional applied flux-drive tone with frequency $\omega_{\mathrm{D}} / 2 \pi=12.944 \mathrm{GHz}$ for different drive powers $\mathbf{P}_{\mathbf{D}}$. The drive power refers to the power at sample input.

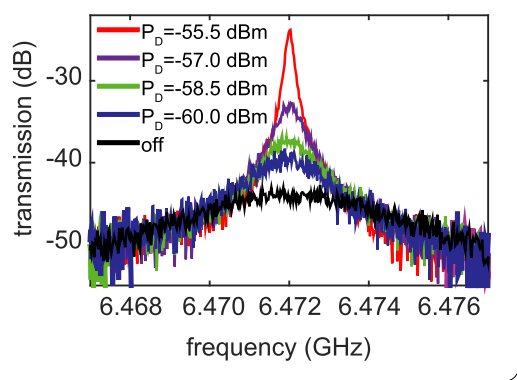

$\Omega_{1} / 2 \pi=6.472 \mathrm{GHz}$ and $\Phi_{\text {ext }}=0.450 \Phi_{0}$ for different values of the drive power. While the gain is increasing for higher drive strength, the bandwidth decreases as expected. For a nondegenerate (phase-insensitive) gain of $G=20 \mathrm{~dB}$, the gain-bandwidth product is $G \Delta \Omega / 2 \pi=21.5 \mathrm{MHz}$. Checking the theoretical relation [48] $\sqrt{G} \Delta \Omega(G)=$ const., we find a maximum deviation of 2.5 similar to other experiments [49].

\section{Competing interests}

The authors declare that they have no competing interests.

\section{Authors' contributions}

The main idea of this paper was proposed by BP and JJGR and, on this basis, the experimental implementation was planned by FD and AM. The sample was fabricated by FW. The experiments were conducted and the data was analyzed by FW with the help of FRK, JG, EPM and FD. The manuscript was mainly prepared by FW with substantial input from KGF, FD, AM and RG. All authors participated in scientific discussions on the results and proofreading of the manuscript. All authors read and approved the final manuscript.

\section{Author details}

${ }^{1}$ Walther-Meißner-Institut, Bayerische Akademie der Wissenschaften, Garching, 85748, Germany. ${ }^{2}$ Physik-Department, Technische Universität München, Garching, 85748, Germany. ${ }^{3}$ Nanosystems Initiative Munich (NIM), Schellingstraße 4 München, 80799, Germany. ${ }^{4}$ Department of Chemistry and Chemical Biology, Harvard University, Cambridge, Cambridge, Massachusetts 02138, United States. ${ }^{5}$ Instituto de Física Fundamental IFF-CSIC, Calle Serrano 113 b, Madrid, 28006, Spain. ${ }^{6}$ Department of Physical Chemistry, University of the Basque Country UPV/EHU, Apartado 644, Bilbao, 48080, Spain. ${ }^{7}$ IKERBASQUE, Basque Foundation for Science, Maria Diaz de Haro 3, Bilbao, 48013, Spain.

\section{Acknowledgements}

The authors acknowledge support from the German Research Foundation through SFB 631 and FE 1564/1-1; the EU projects CCQED, PROMISCE and SCALEQIT; the doctorate program EXQM of the Elite Network of Bavaria; the Spanish MINECO projects FIS2012-33022, FIS2012-36673-C03-02, and FIS2015-69983-P; the CAM Research Network QUITEMAD+; the Basque Government IT472-10 and UPV/EHU UFI 11/55. ES acknowledges support from a TUM August-Wilhelm Scheer Visiting Professorship and hospitality of Walther-Meißner-Institut and TUM Institute for Advanced Study.

\section{Endnotes}

a Of course, the ferromagnetic layer of Ref. [32] was skipped.

$\mathrm{b}$ The values given here and in the following for $\omega_{\mathrm{A}}, \omega_{\mathrm{B}}$ and $\beta$ are obtained by fitting Equation (10) in Section 4 to the data displayed in Figure 2

Received: 14 March 2016 Accepted: 8 July 2016 Published online: 28 July 2016

\section{References}

1. Pierre M, Svensson I-M, Sathyamoorthy SR, Johansson G, Delsing P. Storage and on-demand release of microwaves using superconducting resonators with tunable coupling. Appl Phys Lett. 2014;104(23):232604. doi:10.1063/1.4882646.

2. Strand JD, Ware M, Beaudoin F, Ohki TA, Johnson BR, Blais A, Plourde BLT. First-order sideband transitions with flux-driven asymmetric transmon qubits. Phys Rev B. 2013;87:220505. doi:10.1103/PhysRevB.87.220505.

3. Leek PJ, Filipp S, Maurer P, Baur M, Bianchetti R, Fink JM, Göppl M, Steffen L, Wallraff A. Using sideband transitions for two-qubit operations in superconducting circuits. Phys Rev B. 2009;79:180511. doi:10.1103/PhysRevB.79.180511.

4. Bergeal N, Vijay R, Manucharyan VE, Siddiqi I, Schoelkopf RJ, Girvin SM, Devoret MH. Analog information processing at the quantum limit with a Josephson ring modulator. Nat Phys. 2010;6:296-302. http://dx.doi.org/10.1038/nphys1516.

5. Makhlin Y, Schön G, Shnirman A. Josephson-junction qubits with controlled couplings. Nature. 1999;398(6725):305-7. http://dx.doi.org/10.1038/18613.

6. Baust A, Hoffmann E, Haeberlein M, Schwarz MJ, Eder P, Goetz J, Wulschner F, Xie E, Zhong L, Quijandría F, Peropadre B, Zueco D, García Ripoll J-J, Solano E, Fedorov K, Menzel EP, Deppe F, Marx A, Gross R. Tunable and switchable coupling between two superconducting resonators. Phys Rev B. 2015;91:014515. doi:10.1103/PhysRevB.91.014515. 
7. Niskanen AO, Nakamura Y, Tsai J-S. Tunable coupling scheme for flux qubits at the optimal point. Phys Rev B. 2006;73:094506. doi:10.1103/PhysRevB.73.094506.

8. Niskanen AO, Harrabi K, Yoshihara F, Nakamura Y, Lloyd S, Tsai JS. Quantum coherent tunable coupling of superconducting qubits. Science. 2007;316(5825):723-6. doi:10.1126/science.1141324.

9. Hoi I-C, Kockum AF, Palomaki T, Stace TM, Fan B, Tornberg L, Sathyamoorthy SR, Johansson G, Delsing P, Wilson CM. Giant cross Kerr effect for propagating microwaves induced by an artificial atom. Phys Rev Lett. 2013;111:053601. doi:10.1103/PhysRevLett.111.053601.

10. van der Ploeg SHW, Izmalkov A, van den Brink AM, Hübner U, Grajcar M, Il'ichev E, Meyer H-G, Zagoskin AM. Controllable coupling of superconducting flux qubits. Phys Rev Lett. 2007;98:057004. doi:10.1103/PhysRevLett.98.057004

11. Hime T, Reichardt PA, Plourde BLT, Robertson TL, Wu C-E, Ustinov AV, Clarke J. Solid-state qubits with current-controlled coupling. Science. 2006;314(5804):1427-9. doi:10.1126/science.1134388.

12. Yin Y, Chen Y, Sank D, O'Malley PJJ, White TC, Barends R, Kelly J, Lucero E, Mariantoni M, Megrant A, Neill C, Vainsencher A, Wenner J, Korotkov AN, Cleland AN, Martinis JM. Catch and release of microwave photon states. Phys Rev Lett. 2013;110:107001. doi:10.1103/PhysRevLett.110.107001.

13. Allman MS, Whittaker JD, Castellanos-Beltran M, Cicak K, da Silva F, DeFeo MP, Lecoca F, Sirois A, Teufel JD, Aumentado J, Simmonds RW. Tunable resonant and nonresonant interactions between a phase qubit and $L C$ resonator. Phys Rev Lett. 2014:112:123601. doi:10.1103/PhysRevLett.112.123601.

14. Chen Y, Neill C, Roushan P, Leung N, Fang M, Barends R, Kelly J, Campbell B, Chen Z, Chiaro B, Dunsworth A, Jeffrey E, Megrant A, Mutus JY, O'Malley PJJ, Quintana CM, Sank D, Vainsencher A, Wenner J, White TC, Geller MR, Cleland AN Martinis JM. Qubit architecture with high coherence and fast tunable coupling. Phys Rev Lett. 2014;113:220502. doi:10.1103/PhysRevLett.113.220502.

15. Flurin E, Roch N, Pillet JD, Mallet F, Huard B. Superconducting quantum node for entanglement and storage of microwave radiation. Phys Rev Lett. 2015;114:090503. doi:10.1103/PhysRevLett.114.090503.

16. Leib M, Deppe F, Marx A, Gross R, Hartmann MJ. Networks of nonlinear superconducting transmission line resonators. New J Phys. 2012;14(7):075024. http://stacks.iop.org/1367-2630/14/i=7/a=075024.

17. Hartmann MJ. Polariton crystallization in driven arrays of lossy nonlinear resonators. Phys Rev Lett. 2010;104:113601. doi:10.1103/PhysRevLett.104.113601

18. Grujic T, Clark SR, Jaksch D, Angelakis DG. Repulsively induced photon superbunching in driven resonator arrays. Phys Rev A. 2013;87:053846. doi:10.1103/PhysRevA.87.053846.

19. Gallemí A, Guilleumas M, Martorell J, Mayol R, Polls A, Juliá-Díaz B. Fragmented condensation in Bose-Hubbard trimers with tunable tunnelling. New J Phys. 2015;17(7):073014. http://stacks.iop.org/1367-2630/17/i=7/a=073014

20. Naether U, Quijandría F, García-Ripoll JJ, Zueco D. Stationary discrete solitons in a driven dissipative Bose-Hubbard chain. Phys Rev A. 2015;91:033823. doi:10.1103/PhysRevA.91.033823.

21. Peropadre B, Zueco D, Wulschner F, Deppe F, Marx A, Gross R, García-Ripoll JJ. Tunable coupling engineering between superconducting resonators: from sidebands to effective gauge fields. Phys Rev B. 2013;87(13):134504. doi:10.1103/PhysRevB.87.134504

22. Tian L, Allman MS, Simmonds RW. Parametric coupling between macroscopic quantum resonators. New J Phys. 2008:10:115001. doi:10.1088/1367-2630/10/11/115001

23. van den Brink AM, Berkley AJ, Yalowsky M. Mediated tunable coupling of flux qubits. New J Phys. 2005;7:230. doi:10.1088/1367-2630/7/1/230.

24. Reuther GM, Zueco D, Deppe F, Hoffmann E, Menzel EP, WeißI T, Mariantoni M, Kohler S, Marx A, Solano E, Gross R, Hänggi P. Two-resonator circuit quantum electrodynamics: dissipative theory. Phys Rev B. 2010;81:144510. doi:10.1103/PhysRevB.81.144510

25. Mariantoni M, Deppe F, Marx A, Gross R, Wilhelm FK, Solano E. Two-resonator circuit quantum electrodynamics: a superconducting quantum switch. Phys Rev B. 2008:78:104508. doi:10.1103/PhysRevB.78.104508.

26. Allman MS, Altomare F, Whittaker JD, Cicak K, Li D, Sirois A, Strong J, Teufel JD, Simmonds RW. rf-SQUID-mediated coherent tunable coupling between a superconducting phase qubit and a lumped-element resonator. Phys Rev Lett. 2010;104:177004. doi:10.1103/PhysRevLett.104.177004.

27. Geller MR, Donate E, Chen Y, Fang MT, Leung N, Neill C, Roushan P, Martinis JM. Tunable coupler for superconducting Xmon qubits: perturbative nonlinear model. Phys Rev A. 2015;92:012320. doi:10.1103/PhysRevA.92.012320.

28. Harris R, Berkley AJ, Johnson MW, Bunyk P, Govorkov S, Thom MC, Uchaikin S, Wilson AB, Chung J, Holtham E, Biamonte JD, Smirnov AY, Amin MHS, Maassen van den Brink A. Sign- and magnitude-tunable coupler for superconducting flux qubits. Phys Rev Lett. 2007;98:177001. doi:10.1103/PhysRevLett.98.177001.

29. van der Ploeg SHW, Izmalkov A, van den Brink AM, Hübner U, Grajcar M, Ilichev El, Meyer H-G, Zagoskin AM. Controllable coupling of superconducting flux qubits. Phys Rev Lett. 2007;98:057004. doi:10.1103/PhysRevLett.98.057004

30. Chen Y, Neill C, Roushan P, Leung N, Fang M, Barends R, Kelly J, Campbell B, Chen Z, Chiaro B, Dunsworth A, Jeffrey E, Megrant A, Mutus JY, O'Malley PJJ, Quintana CM, Sank D, Vainsencher A, Wenner J, White TC, Geller MR, Cleland AN, Martinis JM. Qubit architecture with high coherence and fast tunable coupling. arXiv:1402.7367 (2014).

31. Bourassa J, Gambetta JM, Abdumalikov AA, Astafiev O, Nakamura Y, Blais A. Ultrastrong coupling regime of cavity QED with phase-biased flux qubits. Phys Rev A. 2009;80:032109. doi:10.1103/PhysRevA.80.032109.

32. Wild G, Probst C, Marx A, Gross R Josephson coupling and Fiske dynamics in ferromagnetic tunnel junctions. Eur Phys J B. 2010;78(4):509-23. doi:10.1140/epjb/e2010-10636-4.

33. Goetz J, Deppe F, Haeberlein M, Wulschner F, Zollitsch CW, Meier S, Fischer M, Eder P, Xie E, Fedorov KG, Menzel EP, Marx A, Gross R. Loss mechanisms in superconducting thin film microwave resonators. J Appl Phys. 2016;119:015304. doi:10.1063/1.4939299.

34. Sage JM, Bolkhovsky V, Oliver WD, Turek B, Welander PB. Study of loss in superconducting coplanar waveguide resonators. J Appl Phys. 2011;109(6):063915. doi:10.1063/1.3552890.

35. Göppl M, Fragner A, Baur M, Bianchetti R, Filipp S, Fink JM, Leek PJ, Puebla G, Steffen L, Wallraff A. Coplanar waveguide resonators for circuit quantum electrodynamics. J Appl Phys. 2008;104(11):113904. doi:10.1063/1.3010859.

36. Wahl F, Schmidt G, Forrai L. On the significance of antiresonance frequencies in experimental structural analysis. J Sound Vib. 1999;219(3):379-94. doi:10.1006/jsvi.1998.1831. 
37. Sames C, Chibani H, Hamsen C, Altin PA, Wilk T, Rempe G. Antiresonance phase shift in strongly coupled cavity QED. Phys Rev Lett. 2014;112:043601. doi:10.1103/PhysRevLett.112.043601.

38. Houck AA, Tureci HE, Koch J. On-chip quantum simulation with superconducting circuits. Nat Phys. 2012;8(4):292-9. http://dx.doi.org/10.1038/nphys2251.

39. Raftery J, Sadri D, Schmidt S, Türeci HE, Houck AA. Observation of a dissipation-induced classical to quantum transition. Phys Rev X. 2014;4:031043. doi:10.1103/PhysRevX.4.031043.

40. Bell MT, Samolov A. Traveling-wave parametric amplifier based on a chain of coupled asymmetric SQUIDs. Rev Phys Appl. 2015;4:024014. doi:10.1103/PhysRevApplied.4.024014.

41. White TC, Mutus JY, Hoi I-C, Barends R, Campbell B, Chen Y, Chen Z, Chiaro B, Dunsworth A, Jeffrey E, Kelly J, Megrant A, Neill C, O'Malley PJJ, Roushan P, Sank D, Vainsencher A, Wenner J, Chaudhuri S, Gao J, Martinis JM. Traveling wave parametric amplifier with Josephson junctions using minimal resonator phase matching. Appl Phys Lett. 2015;106(24):242601. doi:10.1063/1.4922348.

42. O'Brien K, Macklin C, Siddiqi I, Zhang X. Resonant phase matching of Josephson junction traveling wave parametric amplifiers. Phys Rev Lett. 2014;113:157001. doi:10.1103/PhysRevLett.113.157001.

43. Zhong L, Menzel EP, Di Candia R, Eder P, Ihmig M, Baust A, Haeberlein M, Hoffmann E, Inomata K, Yamamoto T, Nakamura Y, Solano E, Deppe F, Marx A, Gross R. Squeezing with a flux-driven Josephson parametric amplifier. New J Phys. 2013;15(12):125013. http://stacks.iop.org/1367-2630/15/i=12/a=125013.

44. Menzel EP, Di Candia R, Deppe F, Eder P, Zhong L, Ihmig M, Haeberlein M, Baust A, Hoffmann E, Ballester D, Inomata K, Yamamoto T, Nakamura Y, Solano E, Marx A, Gross R. Path entanglement of continuous-variable quantum microwaves. Phys Rev Lett. 2012;109(25):250502. doi:10.1103/PhysRevLett.109.250502.

45. Yamamoto T, Inomata K, Watanabe M, Matsuba K, Miyazaki T, Oliver WD, Nakamura Y, Tsai JS. Flux-driven Josephson parametric amplifier. Appl Phys Lett. 2008;93:042510. doi:10.1063/1.2964182.

46. Bergeal N, Schackert F, Metcalfe M, Vijay R, Manucharyan VE, Frunzio L, Prober DE, Schoelkopf RJ, Girvin SM, Devoret MH. Phase-preserving amplification near the quantum limit with a Josephson ring modulator. Nature. 2010;465:64-8. doi:10.1038/nature09035.

47. Stehlik J, Liu Y-Y, Quintana CM, Eichler C, Hartke TR, Petta JR. Fast charge sensing of a cavity-coupled double quantum dot using a Josephson parametric amplifier. Rev Phys Appl. 2015;4:014018. doi:10.1103/PhysRevApplied.4.014018.

48. Yurke B, Buks E. Performance of cavity-parametric amplifiers, employing Kerr nonlinearites, in the presence of two-photon loss. J Lightwave Technol. 2006;24:5054-66. doi:10.1109/JLT.2006.884490.

49. Castellanos-Beltran MA, Irwin KD, Vale LR, Hilton GC, Lehnert KW. Bandwidth and dynamic range of a widely tunable Josephson parametric amplifier. IEEE Trans Appl Supercond. 2009:19(3):944-7. doi:10.1109/TASC.2009.2018119.

\section{Submit your manuscript to a SpringerOpen ${ }^{\circ}$ journal and benefit from:}

- Convenient online submission

Rigorous peer review

- Immediate publication on acceptance

- Open access: articles freely available online

- High visibility within the field

- Retaining the copyright to your article 